\title{
PROPUESTA DE UNA ARQUITECTURA INFORMÁTICA PARA INTEGRAR LA INFORMACIÓN DE LOS DERECHO-HABIENTES EN UN EXPEDIENTE CLÍNICO ELECTRÓNICO INTEGRAL
}

\author{
Fuentes-Penna Alejandro ${ }^{1}$, Ruiz-Vanoye Jorge ${ }^{2}$ \\ ${ }^{1}$ Escuela Superior de Tlahuelilpan, Universidad Autónoma del Estado de \\ Hidalgo \\ ${ }^{2}$ Universidad Autónoma del Carmen \\ alexfp10@hotmail.com
}

RESUMEN

\begin{abstract}
Esta investigación tiene como objetivo proponer una metodología para integrar la información de los derecho-habientes en un expediente único electrónico, considerando las diferentes instancias donde se puede generar información relacionada con el estado de salud de cada paciente. Se propone la incorporación de la metodología ITIL (administración de servicios tecnológicos) como una estructura para vincular la información que se genera en las diferentes áreas de un centro de salud e integrarla en un expediente único. Con ello, se pueden aplicar diferentes técnicas estadísticas ó de inteligencia artificial para encontrar la relación entre los diferentes factores que pueden contribuir a una enfermedad.
\end{abstract}

PALABRAS CLAVES: ITIL, expediente clínico, expediente clínico electrónico, normas mexicanas.

\section{ABSTRACT}

This research propose a methodology for integrate information from patients in a single electronic file integrated by different information instances provided by preventive medicine, familiar medicine, nursing area, rehabilitation area, and so on. ITIL methodology (Information Technology Infrastructure Library) is a structure to link the information generated in different health center areas. With this, the researchers can apply different statistical techniques or artificial intelligence to find the relationship between different factors that may contribute to prevent different diseases.

KEY WORDS: ITIL, clinical record, electronic clinical record, mexican standards. 


\section{INTRODUCCIÓN}

De acuerdo a la norma NOM-168-SSA1-1998 se define al expediente clínico como: "el conjunto de documentos escritos, gráficos e imagenológicos o de cualquier otra índole, en los cuales el personal de salud, deberá hacer los registros, anotaciones y certificaciones correspondientes a su intervención, con arreglo a las disposiciones sanitarias". A su vez, menciona que es indispensable que los prestadores de servicios médicos de carácter público, social y privado estarán obligados a integrar y conservar el expediente clínico en los términos previstos en la presente Norma (durante 5 años contados a partir de la fecha del último acto médico); los establecimientos, serán solidariamente responsables, respecto del cumplimiento de esta obligación por cuanto hace al personal que preste sus servicios en los mismos, independientemente de la forma en que fuere contratado dicho personal (NOM, 2011 y Sánchez y Ramírez, 2006).

El expediente clínico deberá contener lo siguiente:

1. Datos generales

2. Expediente en consulta externa:

a. Historial clínico.

b. Notas de evolución.

c. Nota de interconsulta.

d. Notas de referencia/ traslado.

3. Notas médicas en urgencias (Villatorio, 2005):
a. Inicial,
b. notas de evolución,
c. notas de referencia/ traslado (en caso de ser necesario),
d. notas de interconsulta (en caso de ser necesario).

4. Notas médicas en hospitalización:
a. De ingreso,
b. historia clínica,
c. notas de evolución,
d. notas de referencia/ traslado,
e. notas pre-operatorias,
f. Nota Pre-anestésica, vigilancia y registro anestésico,
g. nota post-operatoria.
h. Notas de egreso.

5. Reportes del personal profesional, técnico y auxiliar:

a. Hoja de enfermería; 
b. De los servicios auxiliares de diagnóstico y tratamiento.

6. Otros documentos:

a. Cartas de consentimiento bajo información, hoja de egreso voluntario, Hoja de notificación al Ministerio Público, Reporte de causa de muerte sujeta a vigilancia epidemiológica, Notas de defunción y de muerte fetal.

En el año 2010, se aprobó la norma NOM-024-SSA3-2010 donde se establecen los objetivos funcionales y funcionalidades que deberán observar los productos de sistemas de expediente clínico electrónico para garantizar la interoperabilidad, procesamiento, interpretación, confidencialidad, seguridad y uso de estándares y catálogos de la información de los registros electrónicos en salud (NOM, 2011b).

Se hace mención en esta norma que el Sistema Nacional de Salud de México actualmente es alimentado por diversas fuentes de información que no son compartidas, homogéneas, ni utilizadas por otras instancias durante el proceso de atención; y las aplicaciones electrónicas existentes (particularmente las referidas a los expedientes clínicos electrónicos) no se comunican entre sí, pues carecen de estándares, catálogos homogéneos y vocabularios definidos. Por lo que se considera importante disponer de forma inmediata la información médica con acceso desde cualquier lugar del país, con lo cual se permita dar seguimiento, por ejemplo, a pacientes que requieren de alta especialidad, garantizando la veracidad e integridad de la información, así como su seguridad y confidencialidad. El poder integrar la información dispersa, así como apoyar el proceso de investigación médica traerá grandes beneficios al sector salud y a toda la población que acceda a los servicios médicos que proporciona el Estado. La evaluación para cumplir con la norma NOM-024-SSA3-2010, se muestra en ella misma (NOM, 2011b).

\section{Marco teórico}

En Fuentes et al. (2010) se describe a la metodología ITIL (Information Technology Infrastructure Library) como un conjunto de procesos relacionados para la administración y operación de la infraestructura de las tecnologías de información, para promover la provisión de servicios óptimos a los clientes. La metodología ITIL considera un escritorio de servicios (service desk) que concentra las peticiones (tickets) de los usuarios donde se especifican las características y el nivel del servicio priorizándolo con base en el grado de relación que tiene con respecto a la actividad principal de la empresa. Dependiendo del nivel de la petición, ésta se clasifica en incidencia, donde la solución no requiere de personas especializadas; ó problema cuya solución requiere de servicios especializados.

ITIL se basa en una arquitectura de 3 etapas (figura 1) donde el escritorio de servicios se considera un primer filtro en el cual da solución a aquellas peticiones que pueden ser resueltas 
mediante actividades simples. Si el escritorio de servicios no tiene el nivel técnico para dar solución a la petición, se turna la petición a la administración de incidencias, donde se considera que las peticiones requieren de un nivel técnico intermedio y cuya solución requiere de personal con especialidad tecnológica. En caso de que el área de administración de incidencias no pueda dar solución al problema, se turna a la administración de problemas, donde se encuentra el personal con un mayor grado de especialización y conocimiento técnico; en este nivel, la solución requiere de un mayor tiempo de respuesta y el uso de equipo adecuado a la petición (Fuentes et al., 2010) .

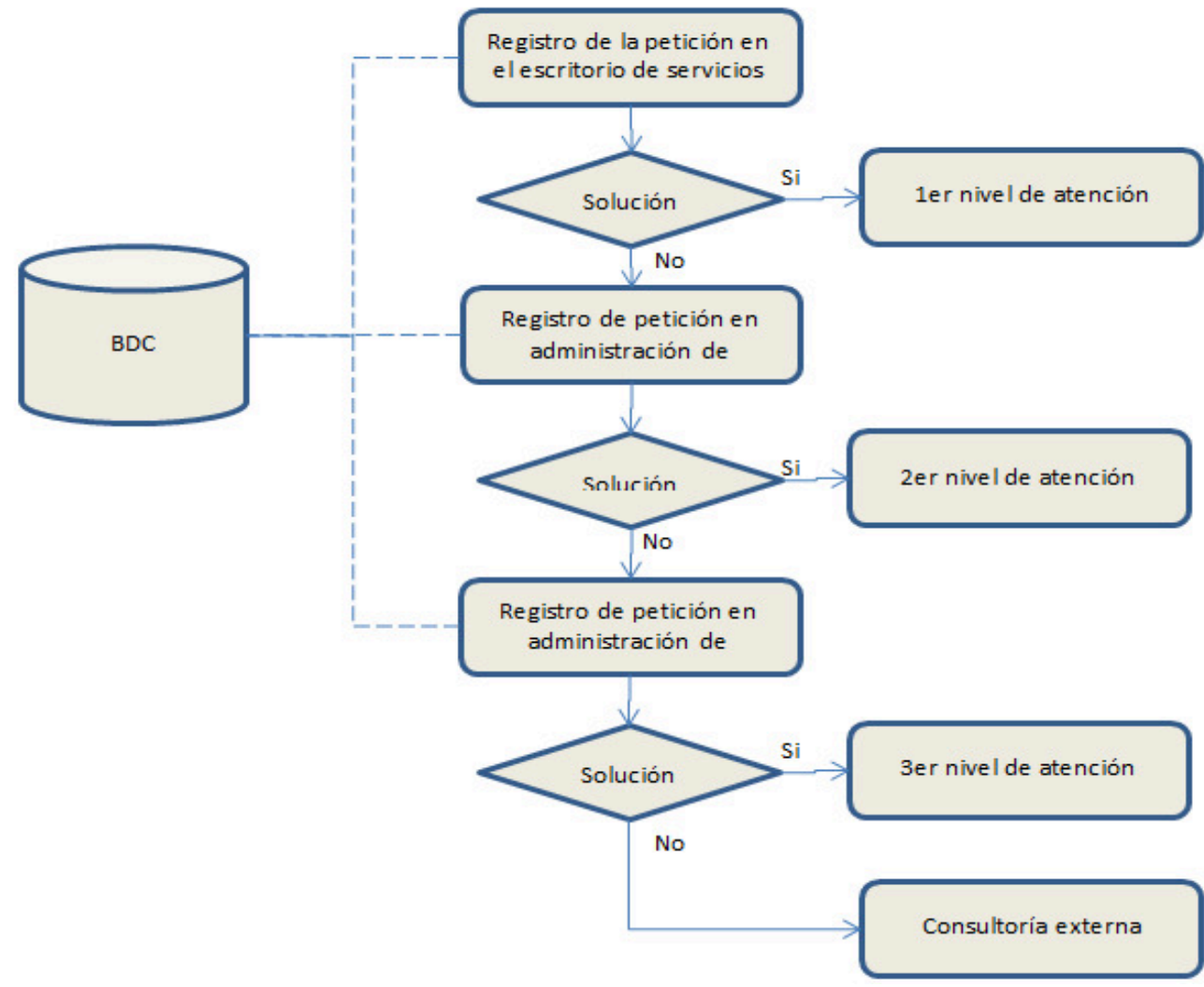

Figura 1. Diagrama de flujo de ITIL

Fuente: Fuentes et al (2010).

Por otra parte, ITIL cuenta con una base de conocimiento donde se almacenan todas las peticiones que se han realizado, las soluciones que se han generado y los tiempos de respuesta; Con esta información, el personal del área de tecnologías de información, tiene una base de datos donde se han registrado todas las incidencias y problemas $\mathrm{y}$, con ello, poder dar solución a 
nuevas peticiones a través de la revisión de soluciones anteriores y del historial que se tiene por equipo de cómputo (Fuentes et al., 2010) .

\section{METODOLOGÍA}

Esta propuesta tiene como objetivo establecer una arquitectura de solución para integrar la información que se genera en las diferentes áreas de un centro de salud e integrar un expediente único que contemple la correlación entre las diferentes áreas del centro.

\section{Arquitectura de la solución}

Se propone el uso de la metodología ITIL como plataforma para integrar las áreas de servicio de un centro de salud conforme a las solicitudes que realizan los derecho-habientes (figura 2).

1. La arquitectura propuesta está dividida en 3 secciones donde se clasifican las áreas del centro de salud:

- Escritorio de servicio: Áreas que son el primer contacto que tienen los derecho-habientes.

- Gestión de incidencias: Áreas que dan servicios médicos generales.

- Gestión de problemas: Áreas médicas que requieren personal con alto grado de especialidad.

2. Base de conocimiento (figura 3): la información que se genera en cada área deberá integrarse en una base de conocimientos única, lo que permitirá realizar operaciones estadísticas, cualitativas y cuantitativas para dar seguimiento a la salud de los pacientes. Se propone integrar una base de conocimiento con los siguientes datos:

\section{Escritorio de servicio:}

1.1. Atención social: esta área deberá fungir como una instancia entrevistadora, siendo el primer filtro en la recepción de información e integrar el expediente clínico del paciente. Se encargará de recopilar los datos correspondientes a:
a. Datos personales
b. Hábitos alimenticios
c. Hábitos de higiene
d. Hábitos deportivos
e. Áreas geográficas donde interactúa
f. Datos laborales
g. Actividades laborales
h. Vicios como cigarro, alcohol, entre otros
i. Relaciones familiares. 
1.2. Recepción: esta área tiene como principales actividades registrar las citas de los derecho-habientes y toma de signos, generando los siguientes datos:

a. Fecha de toma de signos

b. Asunto de la cita

c. Diagnóstico preliminar

d. Signos (temperatura, presión, estatura, peso, entre otros)

1.3. Urgencias: esta área genera información a partir del diagnóstico, evolución del paciente durante su estancia en esta área, tratamiento, notas de interconsulta (caso de ser necesario), notas de traslado (en caso de ser necesario), y aquella información relevante con respecto a la situación del paciente. Los datos que se generan se pueden englobar en:
a. Diagnóstico
b. Notas de evolución del paciente
c. Notas de traslado
d. Notas de traslado
e. Observaciones relevantes

\section{Gestión de incidencias:}

2.1. Medicina preventiva (Aranaz et al, 2011): Esta área se encarga de capturar los datos relacionados con:
a. Interrogatorios relacionados con las campañas, tratamientos preventivos, entre otros
b. Fecha de visita
c. Motivo de la visita
d. Exploración física (en caso de ser necesario)
e. Estudios de laboratorios (en caso de ser necesario)
f. Terapias empleadas (en caso de ser necesario)
g. Diagnóstico de la situación clínica
h. Aplicación de tratamientos como vacunas, dosis orales, etc.

2.2. Medicina general/familiar (Paredes y Rivero, 2001): La información que se genera es a través de las consultas que realizan los médicos responsables de los consultorios, generando los siguientes datos:
a. Interrogatorio (en caso de ser necesario)
b. Exploración física
c. Diagnóstico ó problema clínico
d. Listado de estudios de laboratorios solicitados
e. Terapias solicitadas
f. Tratamientos sugeridos
g. Notas de evolución y/o actualización del cuadro clínico

2.3. Enfermería (Torres et al, 2000): 
a. Fecha de visita

b. Motivo de la visita

c. Tratamiento

d. Doctor a cargo

e. Hoja de enfermería durante la estancia de los pacientes en el hospital: habitus exterior, gráfica de signos vitales tomados en la estancia del paciente y comparándola con los signos de las visitas anteriores, ministración de medicamentos (fecha, hora, cantidad y vía), Procedimientos realizados y observaciones

2.4. Nutrición:
a. Fecha de visita
b. Motivo de la visita
c. Tratamiento sugerido
d. Evolución del paciente
e. Procedimientos realizados
f. Observaciones.

2.5. Vigilancia epidemiológica (Tapia, 1996):
a. Fecha del diagnóstico
b. Periodo de influencia
c. Área de influencia
d. Tratamiento sugerido
e. Posible comportamiento de la epidemia detectada
f. Comportamiento en el cuerpo humano
g. Características de la enfermedad que genera
h. Observaciones.

2.6. Estudios clínicos:
a. Fecha de la solicitud
b. Fecha de entrega
c. Análisis solicitado
d. Nombre del paciente
e. Doctor que solicita el estudio
f. Motivo de la solicitud del estudio
g. Resultados
h. Observaciones.

3. Gestión de problemas:

3.1. Medicina especialidades:

a. Notas de evolució

b. Notas de referencia/ traslado 
3.2. Rehabilitación:
c. Notas pre-operatorias
d. Nota pre-anestésica, vigilancia y registro anestésico
e. Nota post-operatoria
f. Notas de egreso.
a. Fecha de visita
b. Periodo de rehabilitación
c. Motivo de la rehabilitación
d. Doctor responsable de la rehabilitación
e. Doctor que envió al paciente a la rehabilitación
f. Número de sesiones
g. Resultados esperados
h. Descripción de la terapia
i. Evolución del paciente
j. Observaciones.

3.3. Psiquiatría y salud mental:
a. Fecha de visita
b. Motivo de la visita
c. Tipo de visita: individual, grupal, familiar
d. Doctor responsable de sesión
e. Doctor que envió al paciente a la sesión
f. Número de sesiones
g. Resultados esperados
h. Descripción de la sesión
i. Evolución del paciente
j. Observaciones.

\subsection{Control epidemiológico (Tapia, 1996):}

a. Fecha del diagnóstico

b. Periodo de influencia

c. Nombre de la epidemia

d. Causante de la epidemia

e. Comportamiento de la epidemia

f. Áreas de mayor influencia

g. Posible comportamiento de la epidemia detectada

h. Comportamiento en el cuerpo humano

i. Características de la enfermedad que genera

j. Tratamiento sugerido de acuerdo a la evolución de los síntomas

k. Observaciones. 


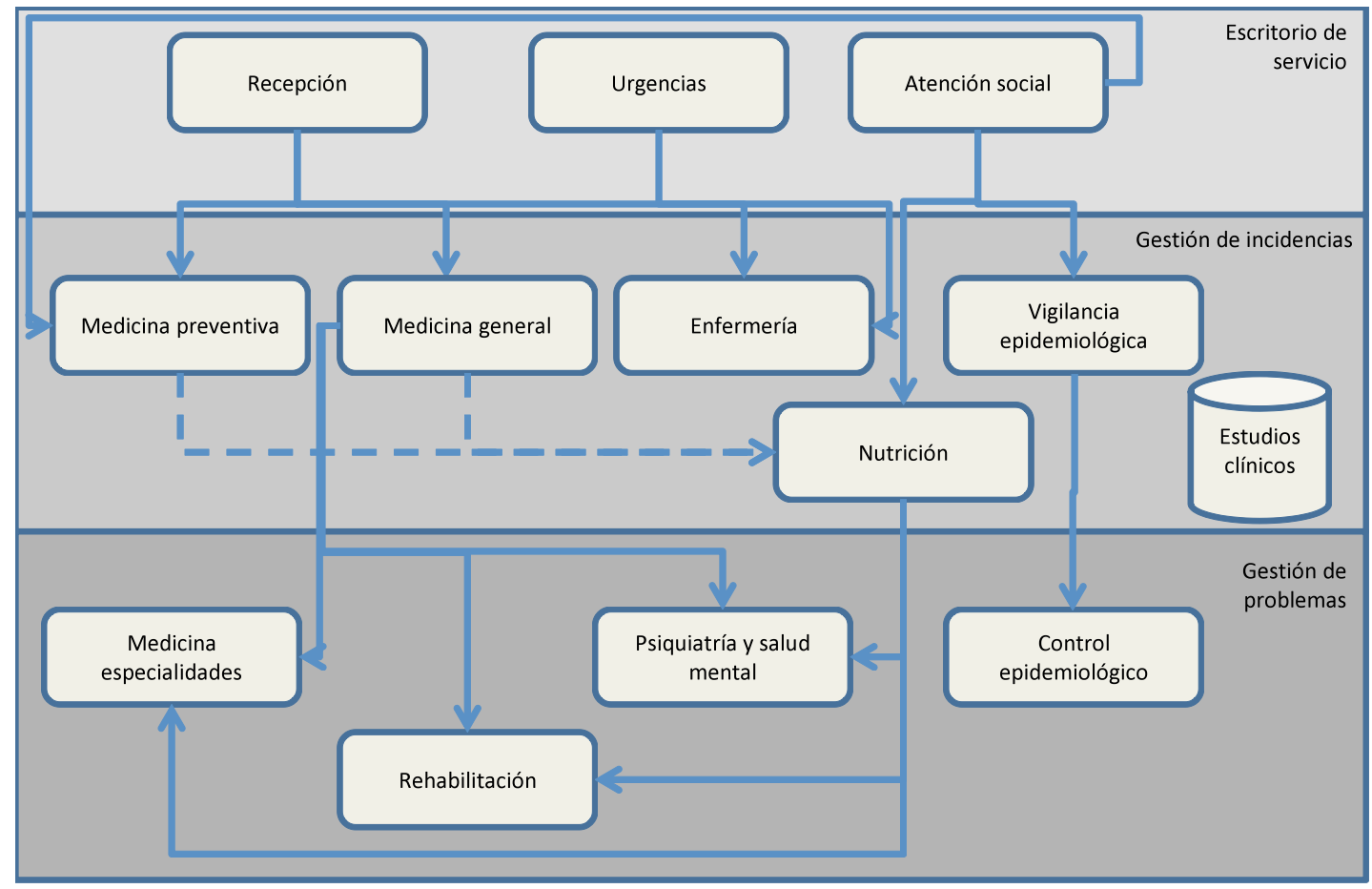

Figura 2. Propuesta de la integración de información

Fuente: Autoría propia basada en Fuentes et al (2010)

Propuesta de solución: En la figura 3 se muestra la base de datos integral, se puede observar que la tabla persona se relaciona indirectamente con todas las tablas a través de la tabla expediente. Con esta propuesta se busca generar un historial clínico integral de todas las visitas que tenga el paciente a las diferentes áreas.

Niveles de seguridad y acceso: Se propone que la seguridad de la información se maneje de forma vertical y horizontal.

$\underline{\text { Vertical: niveles de acceso }}$

- Nivel 1: Visualizar datos restringidos

- Nivel 2: visualizar datos restringidos y agregar nuevos registros que contemplen los datos a los que tiene acceso

○ Nivel 3: Revisión parcial del expediente electrónico del paciente y agregar nuevos registros que contemplen los datos a los que tiene acceso

$\circ \quad$ Nivel 4: Acceso total al expediente, agregar nuevos registros que contemplen los datos a los que tiene acceso y modificar registros que contemplen los datos a los que tiene acceso. 
○ $\quad$ Nivel 5: Acceso total al expediente, permitiendo agregar, modificar y eliminar registros de los expedientes, y en el caso particular, eliminar expedientes.

Horizontal: el acceso a ciertos campos de los registros de información. Se propone un campo de eliminación temporal, es decir, que no se muestren los registros y que se requiera de una confirmación antes de eliminarlos de forma definitiva

\section{Acceso a pacientes:}

Acceso remoto: En este nivel se le permitirá a los pacientes acceder al sistema de información a través del número de paciente expedido en el momento de registrar su expediente médico. Sólo podrá tener acceso a lo siguiente:

- Tratamientos actuales

- $\quad$ Citas programadas

- $\quad$ Próximas campañas de medicina preventiva

- $\quad$ Solicitar nueva cita

Acceso desde el Centro de Salud:

- Datos generales

- $\quad$ Padecimientos

- Tratamientos actuales

- $\quad$ Citas programadas

- $\quad$ Próximas campañas de medicina preventiva

- $\quad$ Solicitar nueva cita

Acceso a las áreas del nivel de escritorio de servicios (únicamente desde el Centro de Salud):

- Programación de citas: En este nivel sólo se permitirá revisar el historial de citas y generar nuevas citas

- Registro de nuevos pacientes

- En el nivel 4 de acceso, se permitirá al área de urgencia revisar el historial clínico de un paciente e ingresar un nuevo registro ligado al expediente a las áreas que pertenecen al nivel de gestión de incidencias para su valoración y, en dado caso, dirigirlo al área de gestión de problemas.

Acceso a las áreas médicas de gestión de incidencias (únicamente desde el Centro de Salud): Las áreas de enfermería y nutrición tendrán acceso nivel 3 (con restricción en los datos generados en nivel de gestión de problemas) para validar los tratamientos que tienen los pacientes actualmente y valorar cada situación; a partir del histórico, estas áreas podrán proponer nuevos tratamientos que mejoren la calidad de vida de los pacientes. 
Las áreas de medicina preventiva, medicina general y vigilancia epidemiológica tendrán acceso de nivel 4 dado que requieren tener acceso al historial completo del paciente para valorar su situación actual y prevenir situaciones que afecten a los pacientes. Si es necesario, podrán canalizar a los pacientes a las áreas de gestión de problemas. Por otra parte, el área de medicina preventiva tendrá acceso a la información relacionada con predicción de escenarios basados en los casos que se han presentado en el centro de salud, en la región y en situaciones anteriores derivadas de las condiciones ambientales.

Acceso a las áreas médicas de gestión de problemas (únicamente desde el Centro de Salud): El área de rehabilitación tendrá nivel de acceso 3, dado que requiere del acceso parcial del expediente clínico del paciente para identificar las causas que han originado el ingreso del paciente a esta área.

El área de psiquiatría y salud mental requiere de este nivel de acceso porque necesitan un panorama general de la situación del paciente para prevenir riesgos a su salud y, por ende, proponer el mejor tratamiento.

Las áreas de especialidades médicas tienen el máximo nivel de acceso, dadas las condiciones de seguridad de la información, ya que involucra el acceso a todo historial médico, teniendo incluso el privilegio de eliminar y modificar información existente

Acceso remoto a las áreas de gestión de incidencias y gestión de problemas: El nivel de acceso remoto sólo permitirá revisar los expedientes que tengan a su cargo de manera actual, ello para dar seguimiento en caso de tener que ausentarse del Centro de Salud. El nivel 3 de acceso remoto es para los médicos cuyo seguimiento de pacientes requiera actualizar notas y actualizar la condición de los pacientes

Acceso a la administración de expedientes: La administración de los expedientes se podrá llevar a cabo a través de la autorización de personal que funja en las áreas de alto nivel del Centro de Salud, dada la responsabilidad que implica el modificar y/o eliminar datos de los expedientes. Al personal autorizado como nivel 5 de administración de expedientes, podrá eliminar los expedientes con el permiso firmado por parte de la persona o, en su caso, por un familiar autorizado. 


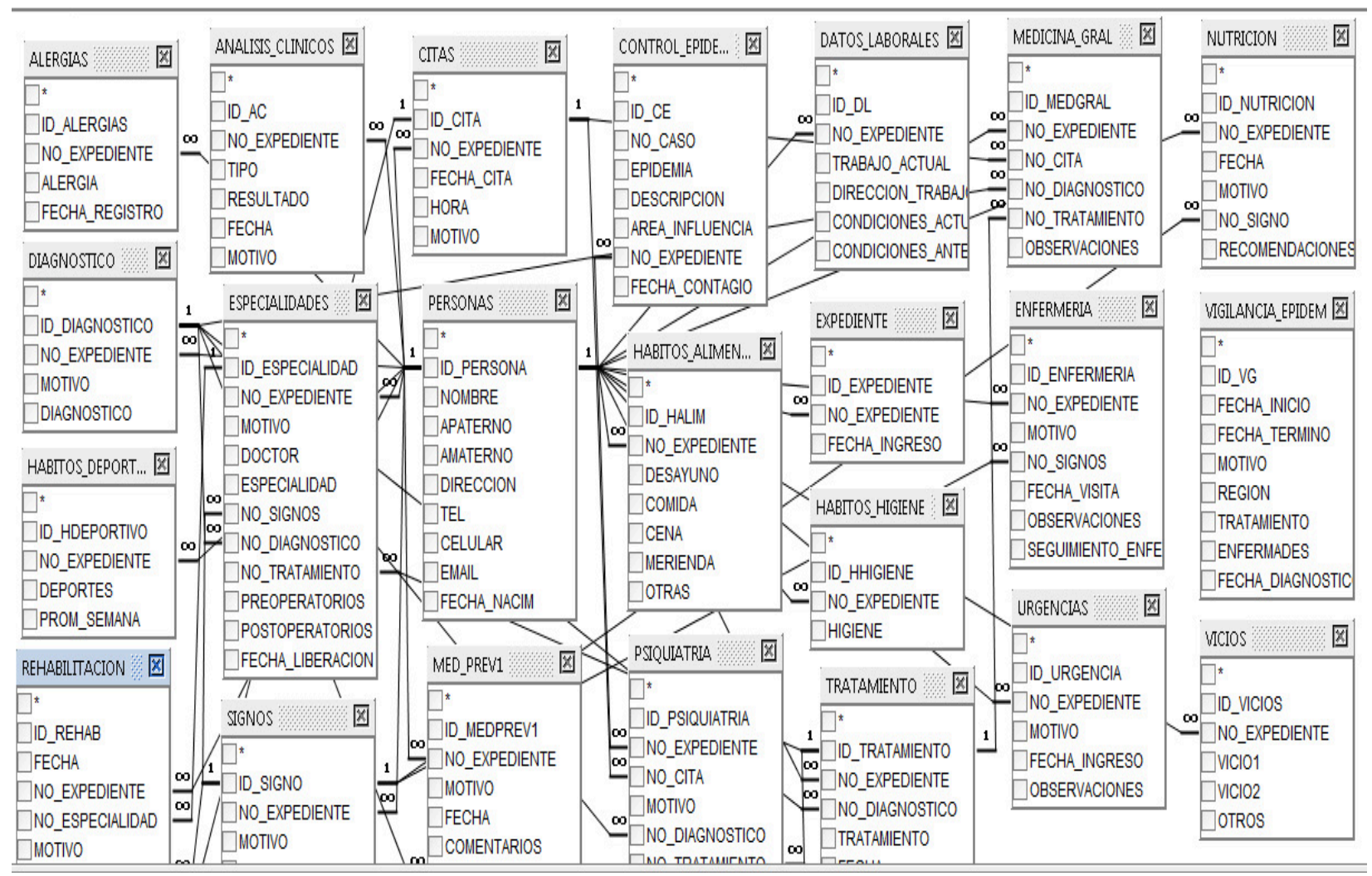

Figura 3. Base de datos

Fuente: Desarrollo propio basado en NOM (2011) y NOM(2011b).

\section{DISCUSIÓN}

Es relevante reunir toda la información que se pueda involucrar con el estado de salud actual de una persona, por lo que se considera como trabajo futuro, complementar la presente investigación con el estudio de diferentes casos donde se han recopilado datos correlacionados con el deterioro de la salud de personas en circunstancias similares.

Por otra parte, es necesario empezar a implementar soluciones como la propuesta de la presente investigación para tener un cúmulo de conocimientos que permitan a los doctores y especialistas de la medicina, generar tratamientos, terapias, etc., que den mejores resultados en relación a mejorar la calidad de vida, bienestar y salud de la población.

En esta propuesta, se plantea el contar con una base de datos que integre todas las áreas del Centro de Salud que generen información del expediente clínico de un paciente con el objetivo de poder relacionar aspectos de salud, higiene, hábitos personales, entre otros, para poder entrelazar esta información y dar un mejor panorama al personal del Centro de Salud. 


\section{CONCLUSIONES}

A partir de la incursión de un Sistema de Información Integral se busca que los pacientes tengan un historial completo de su situación clínica y poder generar una tendencia en cuanto a su salud, identificando los periodos en que su situación de salud se ve afectada. Con ello, cada paciente podrá tomar en cuenta su evolución en aspectos de salud para tomar medidas preventivas.

Por otra parte, las áreas de salud podrán revisar el historial clínico de cada paciente y, en su caso, proporcionar información para prevenir ciertos padecimientos habituales.

Las estadísticas generadas a partir de la información permitirán emplear técnicas como correlación y regresión lineal para predecir el comportamiento de los padecimientos en un periodo anual, incluso, a través del uso de técnicas de minería de datos, encontrar relaciones que, de forma directa, no se visualizan en la detección de situaciones que generen ciertos padecimientos.

\section{REFERENCIAS}

Aranaz, J.M., Pérez, M.V., Mayordomo, C., Navarro, J. F., Tenías, J. M., Segara, L., (...) \& Escolano M. (2011). Cartera de Servicios de Medicina Preventiva y Salud Pública hospitalarios de la Comunidad Valenciana. Sociedad Española de Medicina Preventiva, Salud Pública e Higiene. Valencia: España.

Cruz, A. (2009, 18 de Noviembre). Medicina preventiva exige más que vacunas. Academia Mexicana de Ciencias. Milenio diario.

Fuentes, A., Díaz, O., Zavala, J., Ruiz, J., \& Olivares, J. (2010). Guideline of Identification and Track on Support's level on Mexican Very Small Enterprises. International Journal of Combinatorial Optimization Problems and Informatics. 1(1), 50-55. ISSN: 2007-1558.

González, M. (2005). La investigación esencial en sistemas de salud. Salud Pública de México. Instituto Nacional de Salud Pública 36(4), 408-414 .

Instituto Nacional de Salud Pública (2010). Medicina preventiva: retos de la salud pública en México. Instituto de Salud Pública. Recuperado de http://www.insp.mx/noticias/sistemas-de-salud/1325medicina-preventiva-retos-de-la-salud-publica-en-mexico.html.

Norma Oficial Mexicana (NOM) (2011). NOM-168-SSA1-1998 del expediente clínico. Recuperado de http://www.salud.gob.mx/unidades/cdi/nom/168ssa18.html.

Norma Oficial Mexicana (NOM) (2011b). NOM-024-SSA3-2010. Expediente Clínico. Recuperado de http://dof.gob.mx/nota_detalle.php?codigo $=5158349 \&$ fecha $=08 / 09 / 2010$. 
Organización para la Cooperación y el Desarrollo Económico (2005, 30 de junio). Estudio de la OCDE sobre los Sistemas de Salud - México. Editorial: OECD Publishing . ISBN 9264012427, 9789264012424

Paredes, R. y Rivero, O. (2001). El papel de la medicina general en el sistema nacional de salud. Seminario El ejercicio actual de la medicina. Facultad de Medicina. UNAM. México.

Sánchez-González J., y Ramírez E. (2006). El expediente clínico en México: reflexiones sobre un tema de debate. Rev Mex Patol Clin, 53(3), 166-173.

Secretaría de Salud - Subsecretaría de Innovación y calidad (2007). Programa de acción específico 2007 - 2012. Sistema Nacional de Información de Salud. 1ª Edición. ISBN 978-970-721-5092.

Tapia R. (1996). La importancia de la vigilancia epidemiológica en los servicios de medicina preventiva. Salud Pública de México, 38 (5). Instituto Nacional de Salud Pública.

Torres M., Arina P., Escobar E., López A. \& Palos M. L. (2000). Nursing in the preventive medicine service: functions, activities, responsabilities. Anales del sistema sanitario de Navarra. 23 (2). Vigilancia y control de las infecciones nosocomiales.

Villatorio A. (2005). Definiciones básicas en medicina de urgencia. American British Cowdray Campus Santa Fe. México DF: UMAE General CMN Raza IMSS.

Fecha de recepción: 9 de enero de 2012.

Fecha de aceptación: 16 de marzo de 2013.

Fecha de publicación: 31 de julio del 2013. 